\title{
Knowledge of Rotavirus Disease among Health Care Providers and Their Acceptance of Rotavirus Vaccines in South-East, Nigeria
}

Tagbo Beckie Nnenna ${ }^{1}$, Ughasoro Maduka $D^{2}$, Omotowo Ishola Babatunde ${ }^{3 *}$, Eneh Chizoma Ihuarula ${ }^{4}$ and Uwaezuoke Ndubuisi Anyele

${ }^{1}$ Institute of Child Health, University of Nigeria Teaching Hospital, Enugu, Nigeria

${ }^{2}$ Department of Pediatrics, University of Nigeria, Enugu Campus, PMB 01129 Enugu, Nigeria

${ }^{3}$ Department of Community Medicine, University of Nigeria, Enugu Campus, Nigeria

${ }^{4}$ Department of Pediatrics, University of Nigeria Teaching Hospital, Enugu, Nigeria

\begin{abstract}
Rotavirus is the commonest cause of severe diarrheal disease worldwide, with prevalence as high as $25 \%$ in Nigeria. Severe rotavirus disease is vaccine preventable, with potential to prevent 576 deaths per day in Africa, yet $85 \%$ of deaths occur in Africa and South-East Asia.

Nigeria is yet to adopt the 2009 WHO recommendation of global inclusion of rotavirus vaccine into the national immunization programme. With pre-existing barriers to immunizing Nigerian children; it is relevant to explore the health-care providers knowledge of rotavirus disease and acceptance of the vaccines pre-introduction.

Methods: We explored the knowledge, beliefs and attitudes of 91 health-care providers towards rotavirus disease and vaccines using questionnaire based in-depth interviews.

Results: Most participants were aware of rotavirus disease, especially as a high priority child health issue. Majority knew vaccination was the most effective preventive method; a fifth thought it was good hygiene and treatment with oral rehydration therapy. $53.9 \%$ were conversant with current rotavirus vaccines, but mainly concerned about their newness, yet $70.3 \%$ would vaccinate their children. Majority would recommend vaccination and this likelihood would increase if the vaccine was incorporated into the national immunization programme and recommended by professional organizations. However, only $27.5 \%$ considered rotavirus disease to be a very serious disease and only $63.7 \%$ knew that immunization is the most effective method of prevention. Sustained awareness to enhance uptake was the most frequently expressed need.
\end{abstract}

Conclusion: This study suggests that health-care providers are likely to adopt rotavirus vaccines, especially if they are properly informed. Increasing awareness and involving pediatricians would be essential to a successful vaccination programme. These data, will guide pre-introduction advocacy efforts and help develop strategies to broaden vaccine coverage.

Keywords: Rotavirus disease; Rotavirus vaccines; Health care providers; Knowledge; Acceptance

\section{Introduction}

Rotaviruses are the commonest causes of severe diarrheal disease worldwide [1,2] with prevalence as high as $25 \%$ in Nigeria. Every year Rotavirus causes 111 million cases of illness, 25 million clinic visits and two million hospitalization [3,4]. It is responsible for about 450,000 to 700,000 deaths annually. About $85 \%$ of rotavirus deaths occur in Africa and south East Asia [2] Almost every child will suffer at least one infection before the age of 3 years.

Rotavirus diarrhea has been attributed to several different mechanisms, including malabsorption secondary to enterocyte destruction $[5,6]$, a virus encoded toxin, stimulation of the enteric nervous system (ENS), and villus ischemia [6,7]. New evidence indicates that rotavirus infection is systemic [5]. Rotavirus infection causes a number of changes in the villus epithelium that contribute to malabsorption [7]. Malabsorption results in the transit of undigested mono and disaccharides, carbohydrates, fats, and proteins into the colon. The undigested bolus is osmotically active, and the colon is unable to absorb sufficient water, leading to an osmotic diarrhea [7]. Secretory component of the diarrhea is related to elevated levels of $\mathrm{PGE}_{2}[8]$, toxin-like activity of nonstructural protein (NSP4) [7,9].

In all age groups, the classical clinic presentation is fever and vomiting, followed 2-3 days later by non-bloody diarrhea which can be profuse and lead to severe and life-threatening dehydration. Thus hospitalization and intravenous fluid administration constitute the mainstay of therapy. Some patients are asymptomatic [10], suggesting that both viral and host factors can affect disease severity.

The virus replicates in the non dividing mature enterocytes near the tips of the villi, the pathologic lesions vary between individuals but there is no absolute correlation between these and diseases symptoms $[5,11,12]$. It is challenging to differentiate rotavirus diarrhea and disease from other diarrheal diseases because of multiplicity of infection in children in endemic areas, however, diagnosis of rotavirus can be done by identifying the virus in patient's stool using Enzyme immunoassay (most sensitive for group A rotavirus [13] and recent infections), electron microscopy, polyacrylamide gel electrophoresis and reverse transcriptase polymerase chain reaction [13]. Natural immunity to rotavirus is created by repeated infections.

*Corresponding author: Omotowo Ishola Babatunde, Department of Community Medicine, University of Nigeria, Enugu Campus, PMB 01129 Enugu, Nigeria, Tel: +234-806-581-9918; E-mail: babatundeomotowo@yahoo.com

Received April 30, 2013; Accepted May 20, 2013; Published May 25, 2013

Citation: Tagbo BN, Ughasoro MD, Omotowo BI, Eneh $\mathrm{Cl}$, Uwaezuoke NA (2013) Knowledge of Rotavirus Disease among Health Care Providers and Their Acceptance of Rotavirus Vaccines in South-East, Nigeria. J Vaccines Vaccin S1: 005. doi:10.4172/2157-7560.S1-005

Copyright: ( 2013 Tagbo BN, et al. This is an open-access article distributed under the terms of the Creative Commons Attribution License, which permits unrestricted use, distribution, and reproduction in any medium, provided the original author and source are credited. 
Severe rotavirus disease is vaccine preventable and some vaccines have been proven to be safe and effective in children in both developed and developing countries [1-3]. In 1998 a very effective vaccine, Rotashield, was licensed for use in the USA but was withdrawn in less than one year from the market by the manufacturer due to adverse events following immunization -particularly increased episode of intussusceptions [14]. However in 2006, two new vaccines (Rotarix and Rotateq) were licensed for use following results of large scale clinical trials $[15,16]$.

They are live attenuated, and given orally as 2 or 3 doses, at least 4 weeks apart, the first dose is given by 6 weeks of age, the last dose preferably before 16weeks of age but must be given before 24 weeks of age, contraindications to vaccine use include previous allergy to vaccine or its components, immune deficiency states, intussusceptions, severe fever or acute gastroenteritis. Improved knowledge of Rotavirus disease and increased uptake of these efficacious vaccines would result in better treatment and lower mortality in Rotavirus disease. These two vaccines have been shown to be 90 to $100 \%$ protective against severe rotavirus disease and $74-85 \%$ protective against rotavirus disease of any severity [16]. In 2007, WHO therefore strongly recommended the inclusion of rotavirus vaccination in national immunization programmes of regions where vaccine efficacy data support a significant public Heath impact and where appropriate infrastructure and financing mechanism are available [4]. However, WHO was at that time not prepared to recommend global inclusion of rotavirus vaccine into national immunization programmes "until the full potential of the current rotavirus vaccine has been confirmed in all regions of the world, particularly Asia and Africa".

Subsequently, following results of trials in Asian and African countries, in 2009, the WHO published an update to the 2007 recommendation. In the update, $\mathrm{WHO}$ recommended that rotavirus vaccines for infants should be included in all national immunization programmes [5,17]. Also, in countries where diarrheal deaths account for $10 \%$ or more of mortality among children aged less than 5 years, the introduction of the vaccine is strongly recommended [5].

Many countries have introduced the vaccine into their routine immunization programme. In Nigeria, although the vaccine is yet to be introduced, there are moves by stakeholders to ensure that it is introduced. Being a new vaccine, apparent low level of awareness in the country; previous history of intussusceptions with introduction of Rotashield $[17,18]$, as well as low routine immunization coverage, high child mortality [7] and initial poor acceptance of oral polio vaccine in the country, it is therefore relevant to explore the healthcare providers' knowledge of the disease and acceptance of the vaccine, preintroduction. In addition, no similar study to the knowledge of the authors has been carried out in the country.

Our objectives are therefore to determine the level of knowledge of rotavirus disease among healthcare providers; to assess the potential level of acceptance of the vaccine by health workers and to explore the possible reasons for acceptance or non-acceptance of the vaccine. The data so generated could then be used for advocacy, awareness creation, planning and policy formulation.

\section{Materials and Methods}

We carried out a descriptive study of health care providers practicing in health institutions in the south eastern part of Nigeria, who attend to under five children, to determine the level of their knowledge of rotavirus disease and their acceptance of rotavirus vaccine. The health institutions ranged from primary to tertiary health facilities. The study protocol was reviewed and approved by the Ethical Committee of the University of Nigeria Teaching Hospital Enugu.

After obtaining informed consent, all enrolled participants were administered a questionnaire which focused on demographic characteristics including qualifications, years of practice and age of youngest child. Knowledge and rating of knowledge of rotavirus disease, source of knowledge of rotavirus disease, rating of seriousness of rotavirus disease, knowledge of the preventive methods, mainstay of treatment, current vaccine, acceptance and likelihood of recommending these vaccines. Data analysis was done using the Epi Info software as well as the statistical programme for social science (SPSS).

\section{Results}

Overall, we enrolled 91 health care providers in the study; these had varying levels of educational qualifications, training and experience in addition to broad levels of concerns about vaccine safety issues. Majority were practicing in tertiary health institutions (Figure 1 and 2).

Most providers, $95.6 \%$ had heard of rotavirus, $85.7 \%$ knew that it causes mainly gastroenteritis, $79.1 \%$ felt it was a high priority child health issue but when asked to rate how serious rotavirus disease is in terms of morbidity and mortality on 'a scale of one to five with one being 'not serious at all' and five being 'very serious', only $27.5 \%$ considered rotavirus disease to be very serious, $59.4 \%$ considered it of moderate seriousness (score 3-4) while as low as $6.6 \%$ considered it not serious at all. Forty percent obtained their knowledge of rotavirus from school while only $33 \%$ had theirs from literature. Majority $(63.7 \%)$ knew immunization was the most effective method of preventing rotavirus disease, $20.9 \%$ thought it was good sanitation. Concerning the treatment of rotavirus disease, $62.6 \%$ knew that hospitalization/ intravenous fluid were the mainstay of therapy but as high as $24.2 \%$ thought it was oral rehydration therapy. The self rating of personal knowledge of rotavirus disease among respondents was moderate for $40.6 \%$ of respondents while only $9.9 \%$ had the highest score of five (Figure 3 and 4).

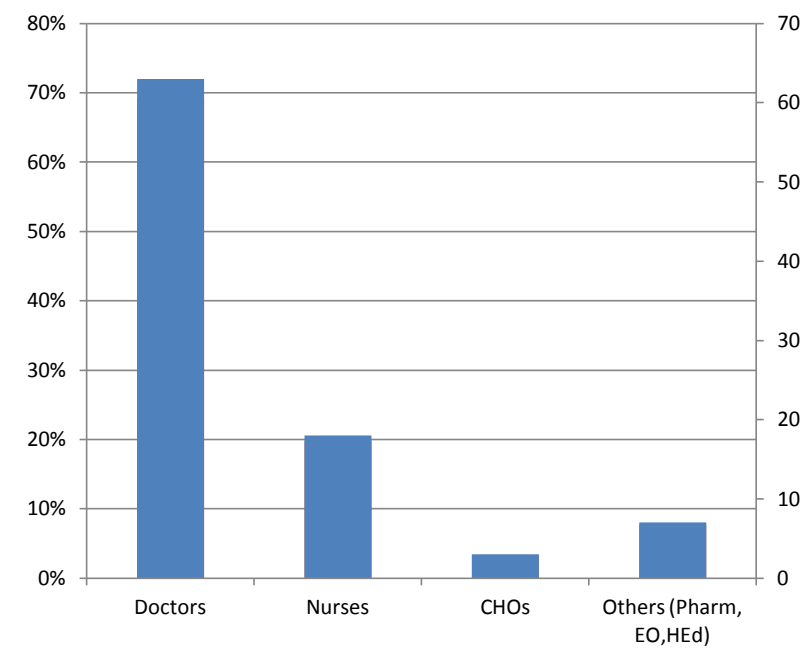

Pham=pharmacist, $\mathrm{EO}=$ environmental health officers, $\mathrm{HEd}=$ health educators

Figure 1: Profession. 
Citation: Tagbo BN, Ughasoro MD, Omotowo BI, Eneh Cl, Uwaezuoke NA (2013) Knowledge of Rotavirus Disease among Health Care Providers and Their Acceptance of Rotavirus Vaccines in South-East, Nigeria. J Vaccines Vaccin S1: 005. doi:10.4172/2157-7560.S1-005

Page 3 of 5

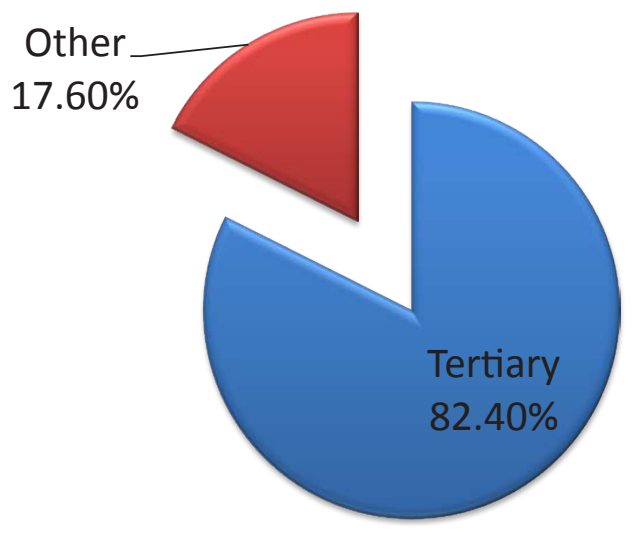

Figure 2: Institution.

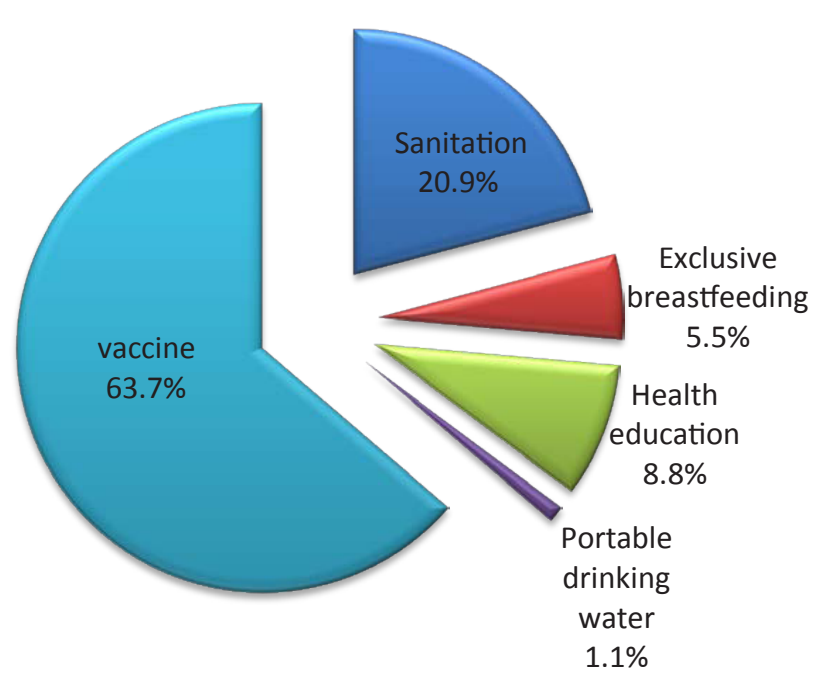

Figure 3: What is the most effective way of prevention?

In vaccine perception, $71.4 \%$ knew that rotavirus vaccines are available but only $45.1 \%$ knew about Rotarix, while a fewer $(14.3 \%)$ knew about Rotashield and (8.8\%) of Rotateq. Fifty eight percent knew these vaccines were live attenuated but as much as $36.6 \%$ didn't know the kind of vaccines these were nor their route of administration. As regards acceptance of these vaccines, $70.3 \%$ would vaccinate their own children with rotavirus vaccine if provided free, only $2.2 \%$ would not (Figure 5).

When asked to rate their likelihood of recommending rotavirus vaccine to their clients following WHO universal recommendation, on a scale of one to five with one being 'not likely at all' and five being 'most likely', the mean score was 4 while only $3.2 \%$ were not likely to recommend rotavirus vaccines. The likelihood of recommendation was likely to increase if the vaccine was incorporated into National Programme on Immunization (NPI) $(72.5 \%)$ or recommended by Pediatric Association of Nigeria (70.3\%) or Federal Ministry of Health (69.2\%) in that order (Figure 6).

Despite these high levels of acceptance for rotavirus vaccines, $29 \%$ respondents expressed concern about the newness of these vaccines (18\%), the 'live' nature of the vaccines and (16\%), safety/efficacy issues. Only 7.7\% were worried about possible adverse events (Figure 7).
Thus, $98.9 \%$ of them think there is need to create awareness about these rotavirus vaccines among health workers. They suggested awareness creation (31.9\%), education of the public (5.5\%), inclusion into NPI as some of the strategies that could lead to increased uptake of the vaccines (Figure 8).

\section{Discussion}

Immunization of infants has been adopted as the primary public health intervention to prevent rotavirus disease for many reasons, such as similar rates in industrialized and less developed countries of the world which indicate that food and environmental hygiene as well as clean water have not significantly decreased the incidence $[19,20]$. Natural infection indicates that initial infection protects against subsequent severe diarrheal disease [21]. Natural immunity was suggested by less frequent episodes and reduced incidence of disease with increasing age [20]. A recent report by the Centre for Disease Control [22] noted that as rotavirus vaccines coverage increased, rotavirus seasons were shorter in durations and diminished in magnitude compared with pre-vaccine seasons.

The qualitative assessment using questionnaire-based interviews of prospective rotavirus vaccines provider provided several valuable insights about potential barriers to immunizing Nigerian children against rotavirus. Such barriers include lack of vaccine awareness, the perception of the risks of vaccination (newness and 'live' nature of vaccine in this instance) as being greater than the usefulness, as well as concerns about efficacy issues. These factors have been cited as major barriers to acceptance of new vaccines [17,23]. The relative lack of basic knowledge reflected by this study is quite significant especially

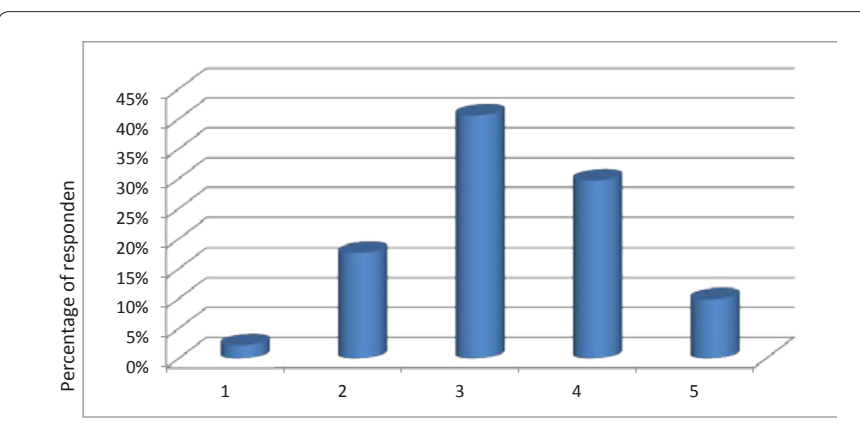

Figure 4: Rate your knowledge of rotavirus.

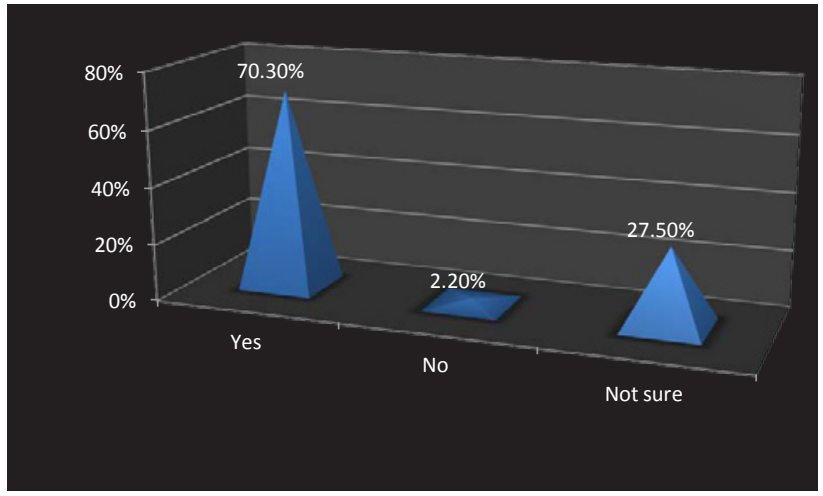

Figure 5: Would you vaccinate your child? 
Citation: Tagbo BN, Ughasoro MD, Omotowo BI, Eneh Cl, Uwaezuoke NA (2013) Knowledge of Rotavirus Disease among Health Care Providers and Their Acceptance of Rotavirus Vaccines in South-East, Nigeria. J Vaccines Vaccin S1: 005. doi:10.4172/2157-7560.S1-005

Page 4 of 5

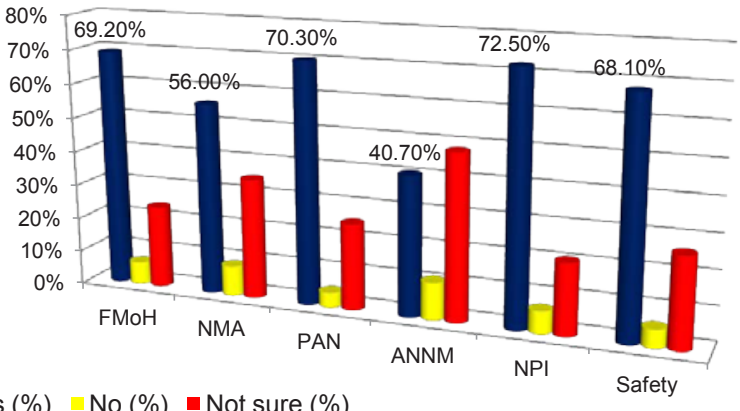

- Yes (\%) No (\%) $\quad$ Not sure $(\%)$

Figure 6: Will likelihood of recommendation increase if recommended by.

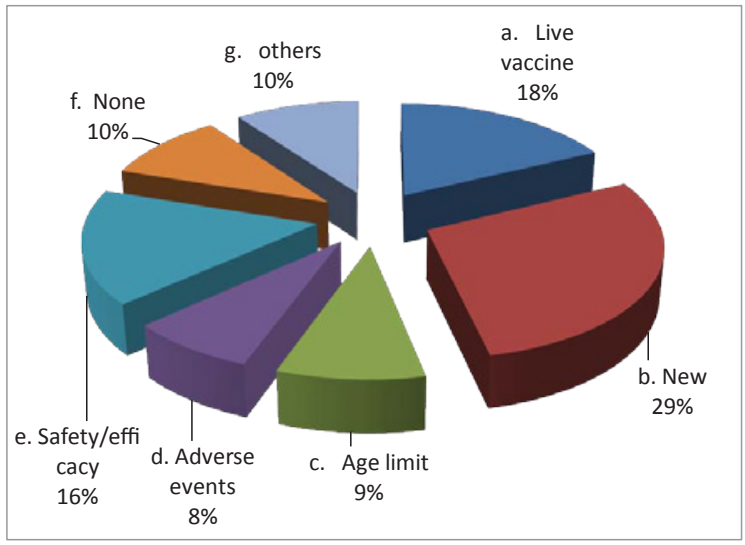

Figure 7: Concern

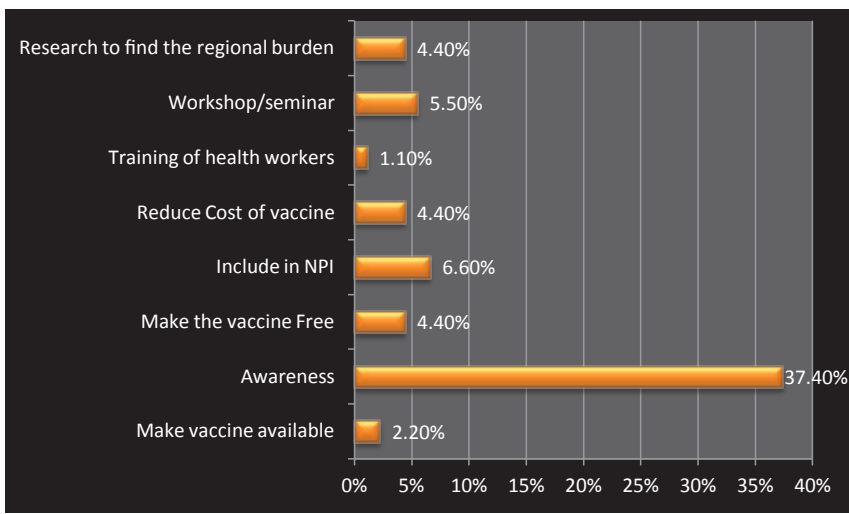

Figure 8: Suggestions.

as many respondents were from tertiary health institutions and many were doctors.

In a national survey among United States pediatricians, possible reluctance of parents to accept the 'new' vaccine (Rotateq) because of safety issues associated with Rotashield was identified as a major potential barrier to acceptance [11]. However, Rotateq has not been associated with intussusceptions or other serious adverse events in large pre-licensure clinical trials $[2,18]$. In the present study, very few expressed concerns about similar adverse events possibly because there was an equally poor knowledge of Rotashield vaccine, moreover the study group is much smaller. While most participants appreciated the health burden of Rotavirus disease and the need for a vaccine (facts which would encourage uptake), a surprisingly high proportion were less aware that vaccination is the most effective way of preventing severe rotavirus disease. Further exploration of the reasons for lack of knowledge of rotavirus vaccine may suggest that the local media used by the public health and medical communities to disseminate information may not be reaching their target audience. In another study, physicians also predicted that the 'live' nature of the vaccine would be a potential barrier to acceptance for some parents [21]. This concern is also reflected in the present study and could be as a result of the fear that a 'live' vaccine may trigger rather than prevent the disease process. Most participants expressed a high likelihood of adopting the new vaccine, particularly if recommended by some professional organizations such as PAN and if incorporated into national programme on immunization (NPI) both of which enjoy high level of confidence in the Nigerian society. The findings in this study is similar to the study conducted by Patel et al. on the qualitative assessment of factors influencing acceptance of a new rotavirus vaccine among healthcare providers [11]. Thus dissemination of information on rotavirus disease and vaccines through these organizations could result in increased vaccine uptake. Further studies would however be necessary to assess this hypothesis; while this provider perspective can serve as additional tool for advocacy to vaccine policy makers.

If the suggestion to create awareness is to be implemented, there would be improvement in acceptance and uptake of these vaccines. This has been shown in a similar study [2] where, upon review of the vaccine information sheet provided by the CDC, more consumers deemed the rotavirus vaccine to be acceptable.

\section{Conclusion/Recommendations}

There is a significant knowledge gap in the population studied. Many health workers would recommend rotavirus vaccines and the likelihood would increase if the vaccine was first recommended by PAN and NPI. This clearly gives a direction on where advocacy should be targeted.

Majority would vaccinate their children if the vaccine is provided free. Efforts to introduce the vaccine into the national free routine immunization programme should be pursued vigorously.

The major concern about the vaccine is that it is new and not the probability of causing intussusceptions. Sustained awareness would therefore address this concern and enhance uptake. Such efforts should start early while plans are ongoing for the introduction of the vaccine. From the results of this study, it would be wise to involve pediatricians to help drive the programme.

\section{References}

1. Rotavirus vaccines (2007) Weekly epidemiological record. World Health Organization 82: 285-296.

2. Simpson E, Wittet S, Bonilla J, Gamazina K, Cooley L, et al. (2007) Use of formative research in developing a knowledge translation approach to rotavirus vaccine introduction in developing countries. BMC Public Heath 7: 281.

3. Dennehy PH (2000) Transmission of rotavirus and other enteric pathogens in the home. Pediatr Infect Dis J 19: S103-105.

4. Conner ME, Ramig RF (1997) Viral enteric disease in Nathanson (editors) Vital Pathogenesis. Lippincott Raven Publishers, Philadelphia 713-743.

5. Ramiq RF (2007) Systemic rotavirus infection. Expert Rev Anti Infect Ther 5 591-612. 
Citation: Tagbo BN, Ughasoro MD, Omotowo BI, Eneh Cl, Uwaezuoke NA (2013) Knowledge of Rotavirus Disease among Health Care Providers and Their Acceptance of Rotavirus Vaccines in South-East, Nigeria. J Vaccines Vaccin S1: 005. doi:10.4172/2157-7560.S1-005

Page 5 of 5

6. Velazques FR, Matson DO, Calva JJ, Guerrero ML, Morrow AL, et al. (1996) Rotavirus infection in infants as protection against subsequent infections. $\mathrm{N}$ Engl Med 335: 1022-1028.

7. Ramig RF (2004) Pathogenesis of intestinal and systemic rotavirus infection. J virol 78: 10213-10220.

8. Rotavirus vaccines: an update (2009) Weekly Epidemiological Record. World Health Organization 84: 533-540.

9. Strategies Advisory Group of Experts (SAGE) (2006) Weekly Epidemiological Record 1: 2-11

10. Meeting of the Strategic Advisory Group of Expertson immunization, October 2009 - conclusions and recommendations (2009) Weekly Epidemiological Record. World Health Organization 84: 517-532.

11. Patel MM, Janssen AP, Tardif RR, Herring M, Parashar UD (2007) A qualitative assessment of factors influencing acceptance of a new rotavirus vaccine among health care providers and consumers. BMC Pediatr 7: 32

12. A qualitative study of health worker and community knowledge, attitude and practices in Kompong Chhang (2002) Attitudes towards immunization in Cambodia 1-45.

13. Cook SM, Glass RI, Lebaron CW, Ho MS (1990) Global seasonality of rotavirus infections. Bull World Health Organ 68: 171-177.

14. Murphy TV, Gargiullo PM, Massoudi MS, Nelson DB, Jumaan AO, et al. (2001) Intussusception among infants given oral rotavirus vaccine. N Engl J Med 344: 564-572.

15. Ruiz-Palacios GM, Perez-Schael I, Velazquez FR, Abate H, Breuer T, et al.
(2006) Safety and efficacy of an attenuated vaccine against severe rotavirus gastroenteritis. N Engl J Med 354: 11-22.

16. Vesikari T, Matson DO, Dennehy P, Van Damme P, Santosham M, et al. (2006) Safety and efficacy of a pentavalent human-bovine (WC3) reassortant rotavirus vaccine. N Engl J Med 354: 23-33.

17. Jiang V, Jiang B, Tate J, Parashar UD, Patel MM (2010) Performance of rotavirus vaccines in developed and developing countries. Hum Vaccin 6: 532542.

18. (2011) A report of Therapeutic Good Administration (TGA's) investigation of a possible safety signal. Rotavirus vaccination and risk of intussusceptions. Australian Government Department of Health and Ageing.

19. Geier DA, King PG, Sykes LK, Geier MR (2012) The temporal relationship between Rotateq immunization and intussusceptions adverse events in the Vaccine Adverse Event Reporting System (VAERS). Med Sci Monit 18: PH1217 .

20. Atherly D, Dreibelbis R, Parashar UD, Levin C, Wecker J, et al. (2009) Rotavirus vaccination: cost-effectiveness and impact on child mortality in developing countries. J Infect Dis 200: S28-38.

21. Bishop RF (1996) Natural history of human rotavirus infection. Arch Virol Supp 12: $119-128$

22. Patel MM, Parashar UD (2009) Assessing the effectiveness and public health impact of rotavirus vaccines after introduction in immunization programs. J Infect Dis 200: S291-299.

23. Iwamoto M, Saari TN, McMahon SR, Yusuf HR, Massoudi MS, et al. (2003) A survey of pediatricians on the reintroduction of a rotavirus vaccine. Pediatrics 112: e6-10.
This article was originally published in a special issue, Tumor Immunology Vaccines handled by Editor(s). Dr. Robert John Amato, The University of Texas, USA 